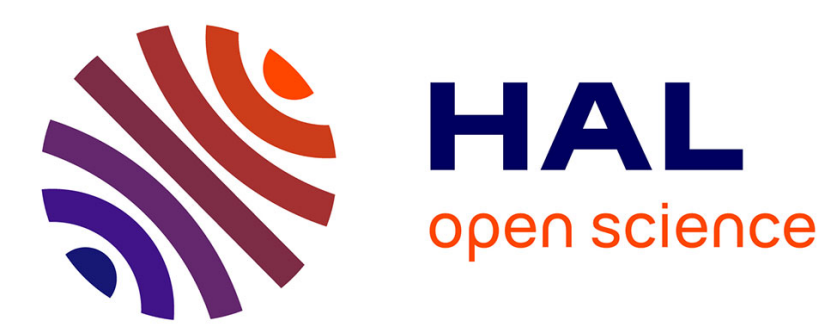

\title{
The Potential Scope of the Ultrasonic Surface Reflection Method Towards Mechanical Characterisation of Isotropic Materials. Part 1. A Theoretical Analysis
}

Violaine Tinard, Pierre Francois, Christophe Fond

\section{- To cite this version:}

Violaine Tinard, Pierre Francois, Christophe Fond. The Potential Scope of the Ultrasonic Surface Reflection Method Towards Mechanical Characterisation of Isotropic Materials. Part 1. A Theoretical Analysis. Experimental Mechanics, 2021, 10.1007/s11340-021-00730-9 . hal-03412188

\author{
HAL Id: hal-03412188 \\ https://hal.science/hal-03412188
}

Submitted on 2 Nov 2021

HAL is a multi-disciplinary open access archive for the deposit and dissemination of scientific research documents, whether they are published or not. The documents may come from teaching and research institutions in France or abroad, or from public or private research centers.
L'archive ouverte pluridisciplinaire HAL, est destinée au dépôt et à la diffusion de documents scientifiques de niveau recherche, publiés ou non, émanant des établissements d'enseignement et de recherche français ou étrangers, des laboratoires publics ou privés. 
The potential scope of the ultrasonic surface reflection method towards mechanical characterisation of isotropic materials. Part 1. A theoretical analysis

V. Tinard ${ }^{\text {a }}$ corresponding author, e-mail: vtinard@ unistra.fr, phone: +33

(0)368858739, fax: +33(0)368858608)

P. François ${ }^{\mathrm{a}}$ (pierre.francois@ unistra.fr)

C. Fond ${ }^{\mathrm{a}}$ (christophe.fond@ unistra.fr)

a ICube, Université de Strasbourg, CNRS UMR 7357, 300 bd Sébastien Brant - CS 10413 - F-67412 ILLKIRCH Cedex, FRANCE

\section{AUTHORS CONTRIBUTIONS}

All authors contributed to the study conception and design. Material preparation, data collection and analysis were performed by Violaine Tinard, Pierre François and Christophe Fond. The first draft of the manuscript was written by Violaine Tinard and all authors commented on previous versions of the manuscript. All authors read and approved the final manuscript.

\section{KEYWORDS}

Ultrasonic methods, Measurement error, Viscoelasticity, Surface Reflection Method. 


\section{ABSTRACT}

Background: This paper deals with the possible field of application of ultrasonic Surface Reflection Method (SRM) to achieve the mechanical characteristics of isotropic materials. This method is based on the measurement of the amplitude of the reflected wave at the interface between reference material and the material to be characterised. Objective: The purpose of Part 1 of this paper is to establish the theoretical conditions for the applicability of SRM. Methods: First, the theoretical formulas necessary to obtain the mechanical properties of the material to be tested will be established. Then, on the basis of these analytical formulas, the validity of the results for the material to be studied will be discussed according to the choice of the mechanical properties of the reference material through uncertainty calculations. The measurand error of SRM is then compared to that of traditional methods (transmission, transmission in water bath, pulse-echo). Results: The analytical solution to the inverse problem (the mechanical characteristics of the tested medium based on those of the reference medium and the waves' amplitude) will be given. From this analytical solution, an analysis of the measurand error will be performed and a method for choosing the reference material will be proposed. Conclusions: It appears that SRM is better suited than traditional methods in two specific cases: measurement of small deviations of mechanical properties from a reference material or characterisation of high damping materials. In Part 2 of this paper, the practical conditions of applicability of the method are described and then applied to different kinds of materials. 


\section{INTRODUCTION}

Ultrasonic methods are commonly used to determine the high-frequency mechanical properties of materials $[1,2]$. Most ultrasonic methods are based on measurements of amplitude and time-of-flight of ultrasonic waves through a sample of known thickness and density. From these two measurements are deduced the celerity and attenuation of ultrasonic waves in the considered medium, which makes it possible to calculate the mechanical moduli (shear and $\mathrm{P}$-wave moduli using respectively transverse and longitudinal waves).

This principle can be implemented using various techniques illustrated in figure 1 . In the transmission mode, two transducers (one emitter and one receiver) are placed face to face on both sides of the sample [3-8]. This method is quite easy to implement but has some disadvantages. It requires access to both sides of the material to be tested, which may not be possible for in-situ measurements. Moreover the precise determination of attenuation often requires several sample thicknesses (in order to avoid the influence of transmission coefficients at interfaces between transducers and material) and appropriate consideration of the ultrasonic beam geometry. The latter problem can be addressed by using transmission mode in water bath [9-13]: it is sufficient in this case to make measurements with and without a sample in order to determine accurately the attenuation coefficient. Another advantage of this method is that the transmission of longitudinal and transverse waves can be studied using the same couple of pressure transducers by simply rotating the sample. However, this method is obviously reserved for laboratory tests. A third method is more suitable for some in-situ tests in which only one side of the material to be tested is accessible. This method, called pulse-echo mode [14-18], measures the transit time and wave amplitude after reflection on the opposite 
side and only requires a single transducer. However, this method has some limitations. If the sample is too thin, the echo may overlap with the sensor's emission signal. Conversely, if the sample is too thick, the amplitude of the reflected signal may be too small to be accurately measured.

Whichever of the three methods outlined above is used, the thickness of the sample must be precisely known, which is not always possible in the context of in-situ measurements. Above all, the main limitation of those three methods is that once the signal has passed through the material its amplitude has to be measurable. Ultrasonic measurement of the mechanical properties of high damping materials may therefore be impossible $[1,19]$.

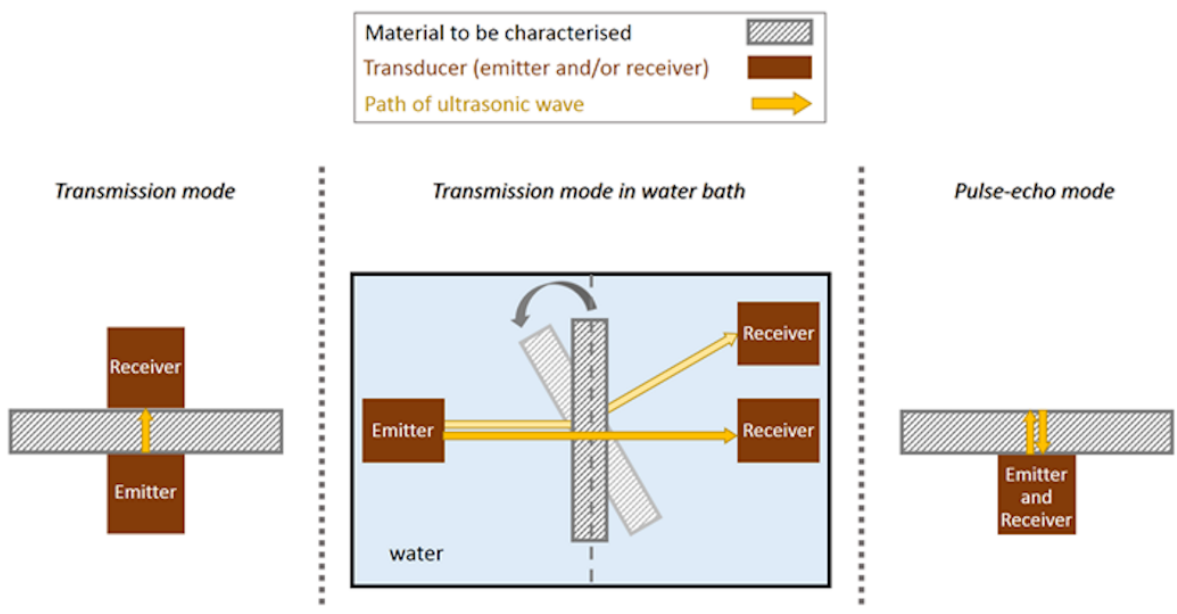

Figure 1. Illustration of the main ultrasonic measurement modes.

However, a fourth method, presented in [20, 21], has been developed to overcome some of these drawbacks: the Surface Reflection Method (SRM). This method is founded on the influence of an interface change on the reflected acoustic wave. In other words, it is based on the measurement of the ratio of the amplitude of the reflected wave at the 
interface between two materials to the amplitude of the reflected wave at the air interface. These amplitudes depend on the mechanical properties of each medium. If one of the media (named in the following reference material) is known, this measurement makes it possible to determine the mechanical characteristics of the second. In this method, the incidence of the emitted pulse may be normal or oblique [22-25]. The measurement accuracy is increased for oblique incidence, at the expense of simplicity of implementation. Only the normal incidence method is considered in this study. A scheme illustrating how the method works is presented in figure 2.

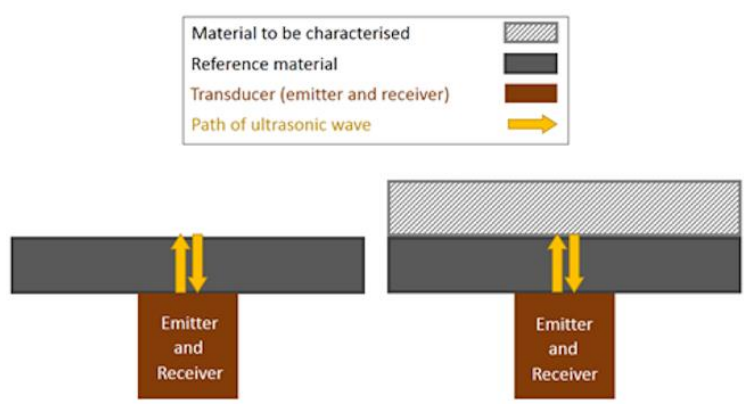

Figure 2. Illustration of the Surface Reflection Method (SRM).

Given the limitations of the traditional methods described previously, the conditions for the applicability of the SRM is discussed below. First, this paper focuses on the theoretical formulation of the amplitude of reflected waves at the interface between two viscoelastic media according to the latter's mechanical characteristics. Subsequently, the analytical solution to the inverse problem (the mechanical characteristics of the tested medium based on those of the reference medium and the waves' amplitude) is given. From this analytical solution, an analysis of the measurand error is performed and a method for choosing the reference material is proposed. 


\section{THEORY}

2.1 Ultrasonic wave propagation in viscoelastic medium

The propagation of a mechanical wave in a continuous and homogeneous medium is governed by Euler's equation:

$$
\nabla \sigma^{*}=\rho a^{*}
$$

Where $\sigma^{*}$ is the complex Cauchy stress, $\rho$ is the density of the material, $a^{*}$ is the complex acceleration and $\nabla$ is the Laplacien operator.

The mechanical behaviour of the material is assumed to be linear viscoelastic in the small strain domain with a complex descriptor denoted below by $\mathrm{D}^{*}$ with real and imaginary parts respectively denoted as $\mathrm{D}^{\prime}$ and $\mathrm{D}^{\prime \prime}$. In case of transverse waves (respectively longitudinal waves) $\mathrm{D}^{*}$ represents the complex shear modulus $\mathrm{G}^{*}$ (respectively the complex $\mathrm{P}$-wave modulus $\mathrm{M}^{*}=\mathrm{K}^{*}+4 \mathrm{G}^{*} / 3$, with $\mathrm{K}^{*}$ the complex bulk modulus). In the case of a plane wave propagating in the $\mathrm{x}$-direction, the complex stress is expressed according to equation 2.

$$
\sigma^{*}=\mathrm{D}^{*} \frac{\partial \mathrm{u}^{*}}{\partial \mathrm{x}}
$$

Where $\mathrm{u}^{*}$ represents the complex displacement.

From equations 1 and 2 the well-known relationship of propagation of mechanical waves in a continuous medium is obtained [26].

$$
\mathrm{D}^{*} \frac{\partial^{2} \mathrm{u}^{*}}{\partial \mathrm{x}^{2}}=\rho \frac{\partial^{2} \mathrm{u}^{*}}{\partial \mathrm{t}^{2}}
$$

In a semi-infinite medium, the stationary solution of equation 3 in case of a harmonic wave of pulsation $\omega$ is expressed: 


$$
u^{*}(t, x)=u_{0} \exp (-\alpha x) \exp \left(j\left(\omega\left(t-\frac{x}{c}\right)\right)\right)
$$

Where $\mathrm{u}_{0}$ is the amplitude of displacement at $\mathrm{x}=0, \alpha$ is the attenuation coefficient, $\omega$ is the wave pulsation and $\mathrm{c}$ is the wave phase celerity.

By substituting the solution expressed in equation 4 in equation 3, the relationship between the real and imaginary part of $\mathrm{D}^{*}$ on the one hand and attenuation and celerity on the other hand has been revealed (equations 5 and 6). This system of equations establishes the method for determining mechanical properties by ultrasonic transmission (figure 1).

$$
\begin{aligned}
& \left\{\begin{array}{l}
\alpha=\frac{\omega \sqrt{\rho} \sqrt{\left|\mathrm{D}^{*}\right|-\mathrm{D}^{\prime}}}{\sqrt{2}\left|\mathrm{D}^{*}\right|} \\
\mathrm{c}=\frac{\sqrt{2}\left|\mathrm{D}^{*}\right| \sqrt{\left|\mathrm{D}^{*}\right|-\mathrm{D}^{\prime}}}{\mathrm{D}^{\prime \prime} \sqrt{\rho}}
\end{array}\right. \\
& \left\{\begin{array}{l}
\mathrm{D}^{\prime}=\rho c^{2} \frac{1-(\alpha \mathrm{c} / \omega)^{2}}{\left(1+(\alpha \mathrm{c} / \omega)^{2}\right)^{2}} \\
\mathrm{D}^{\prime \prime}=\rho \mathrm{c}^{2} \frac{2 \alpha \mathrm{c} / \omega}{\left(1+(\alpha \mathrm{c} / \omega)^{2}\right)^{2}}
\end{array}\right.
\end{aligned}
$$

2.2. Reflection at the interface of two viscoelastic media

Many authors have studied the mechanisms of reflection and transmission of plane waves at the interface between two elastic or viscoelastic media [27-29]. Unlike these authors whose aim was to investigate the propagation of waves at the interface of two known materials, this study focuses on the inverse problem and its analytical solution. 


\subsubsection{Acoustic impedance}

In the present paper, only the case of a normal incidence is considered. When an ultrasonic wave reaches the interface between two media, it is partially reflected and transmitted. The amplitudes of transmitted (denoted $\left.u_{t}{ }^{*}\right)$ and reflected (denoted $u_{r}{ }^{*}$ ) waves depend upon the acoustic impedances of the two media in contact and are proportional to the amplitude of the incident wave (denoted $u_{i}{ }^{*}$ ) as illustrated in figure 3. In what follows, subscript 1 is related to the medium of incident wave and subscript 2 to the secondary medium.

The acoustic impedance $\mathrm{Z}^{*}$ for a viscoelastic medium is defined by equation 7 [26].

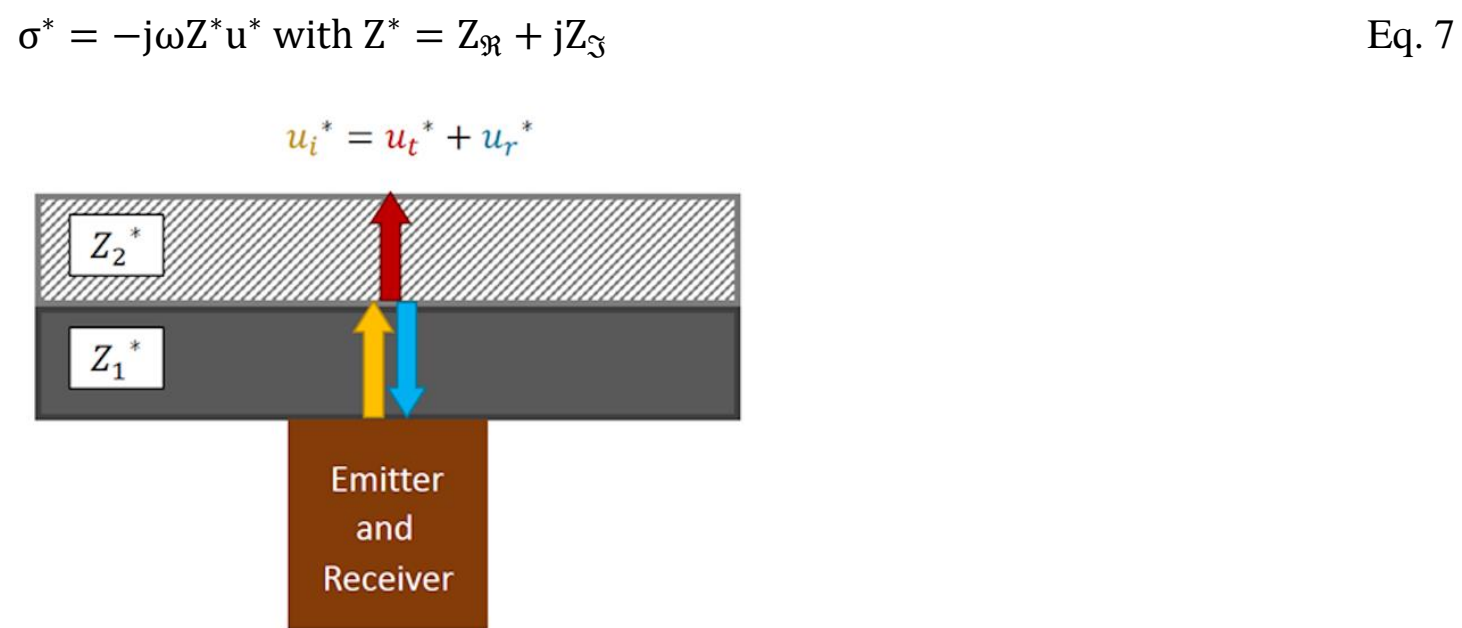

Figure 3. Scheme illustrating the wave propagation through a two-layer medium.

From equations 2 and 7 expressions of real and imaginary parts of $\mathrm{Z}^{*}$ in relation to $\mathrm{D}^{*}$ and $\rho$ are obtained (equation 8). 


$$
\mathrm{D}^{*}=\frac{\mathrm{Z}^{* 2}}{\rho} \Leftrightarrow\left\{\begin{array}{l}
\mathrm{Z}_{\Re}=\sqrt{\frac{\rho}{2}} \frac{\mathrm{D}^{\prime \prime}}{\sqrt{\left|\mathrm{D}^{*}\right|-\mathrm{D}^{\prime}}} \\
\mathrm{Z}_{\mathfrak{I}}=\sqrt{\frac{\rho}{2}} \sqrt{\left|\mathrm{D}^{*}\right|-\mathrm{D}^{\prime}}
\end{array}\right.
$$

Eq. 8

\subsubsection{Conservation equation at the interface}

Displacement and stress continuity at the interface lead to the following set of equations:

$$
\left\{\begin{array}{l}
u_{\mathrm{i}}{ }^{*}=\mathrm{u}_{\mathrm{t}}{ }^{*}+\mathrm{u}_{\mathrm{r}}{ }^{*} \\
\sigma_{\mathrm{i}}{ }^{*}=\sigma_{\mathrm{t}}{ }^{*}+\sigma_{\mathrm{r}}{ }^{*}
\end{array}\right.
$$

By substituting the expression of stress in function of the complex impedance (equation 7), equation 9 leads to:

$$
\left\{\begin{array}{c}
\mathrm{u}_{\mathrm{i}}^{*}=\mathrm{u}_{\mathrm{t}}{ }^{*}+\mathrm{u}_{\mathrm{r}}{ }^{*} \\
\mathrm{z}_{1}{ }^{*} \mathrm{u}_{\mathrm{i}}{ }^{*}=\mathrm{z}_{2}{ }^{*} \mathrm{u}_{\mathrm{t}}{ }^{*}-\mathrm{z}_{1}{ }^{*} \mathrm{u}_{\mathrm{r}}{ }^{*}
\end{array}\right.
$$

Which solution is:

$$
\left\{\begin{array}{l}
\mathrm{u}_{\mathrm{r}}^{*}=\frac{\mathrm{Z}_{2}^{*}-\mathrm{Z}_{1}^{*}}{\mathrm{Z}_{2}^{*}+\mathrm{Z}_{1}^{*}} \mathrm{u}_{\mathrm{i}}^{*} \\
\mathrm{u}_{\mathrm{t}}^{*}=\frac{2 \mathrm{Z}_{1}^{*}}{\mathrm{Z}_{2}^{*}+\mathrm{Z}_{1}^{*}} \mathrm{u}_{\mathrm{i}}^{*}
\end{array}\right.
$$

In what follows, equations are expressed in terms of ratios $\mathrm{U}_{\mathrm{r}}^{*}$ and $\mathrm{U}_{\mathrm{t}}^{*}$ with

$$
\left\{\begin{array}{l}
\mathrm{U}_{\mathrm{r}}^{*}=\mathrm{u}_{\mathrm{r}}{ }^{*} / \mathrm{u}_{\mathrm{i}}{ }^{*} \\
\mathrm{U}_{\mathrm{t}}^{*}=\mathrm{u}_{\mathrm{t}}{ }^{*} / \mathrm{u}_{\mathrm{i}}{ }^{*}
\end{array}\right.
$$

The real and imaginary parts of the amplitude of transmitted and reflected waves are given by equation 13 . 


$$
\left\{\begin{array}{c}
\mathrm{U}_{\mathrm{r} \Re}=\frac{\left|\mathrm{Z}_{2}{ }^{*}\right|^{2}-\left|\mathrm{Z}_{1}{ }^{*}\right|^{2}}{\left|\mathrm{Z}_{1}{ }^{*}+\mathrm{Z}_{2}{ }^{2}\right|^{2}} \\
\mathrm{U}_{\mathrm{r} \mathfrak{I}}=\frac{2\left(\mathrm{Z}_{\Re 1} \mathrm{Z}_{\mathfrak{\Im} 2}-\mathrm{Z}_{\Re 2} \mathrm{Z}_{\mathfrak{I} 1}\right)}{\left|\mathrm{Z}_{1}{ }^{*}+\mathrm{Z}_{2}{ }^{*}\right|^{2}} \\
\mathrm{U}_{\mathrm{t} \Re}=1-\mathrm{U}_{\mathrm{r} \Re} \\
\mathrm{U}_{\mathrm{t} \mathfrak{I}}=-\mathrm{U}_{\mathrm{r} \mathfrak{I}}
\end{array}\right.
$$

Since SRM is based on echo measurements, only the reflected wave is of interest. By inverting the system it is possible to obtain the complex impedance components of medium 2 knowing those of medium 1 and by measuring the reflected wave's amplitude (equation 14).

$$
\left\{\begin{array}{l}
\mathrm{Z}_{\Re 2}=-\frac{\mathrm{Z}_{\Re 1}\left(\mathrm{U}_{\mathrm{r} \Re}{ }^{2}+\mathrm{U}_{\mathrm{r} \mathfrak{I}}{ }^{2}-1\right)+2 \mathrm{Z}_{\mathfrak{I} 1} \mathrm{U}_{\mathrm{r} \mathfrak{I}}}{\left(\mathrm{U}_{\mathrm{r} \Re}-1\right)^{2}+\mathrm{U}_{\mathrm{r} \mathfrak{I}}{ }^{2}} \\
\mathrm{Z}_{\mathfrak{\Im} 2}=-\frac{\mathrm{Z}_{\mathfrak{J} 1}\left(\mathrm{U}_{\mathrm{r} \Re}{ }^{2}+\mathrm{U}_{\mathrm{r} \mathfrak{I}}{ }^{2}-1\right)+2 \mathrm{Z}_{\mathfrak{R} 1} \mathrm{U}_{\mathrm{r} \mathfrak{I}}}{\left(\mathrm{U}_{\mathrm{r} \Re}-1\right)^{2}+\mathrm{U}_{\mathrm{r} \mathfrak{I}}{ }^{2}}
\end{array}\right.
$$

The aim of the method exposed in the present paper is to determine the modulus of medium 2. By substituting in equation 14 the impedance components using their expressions in function of the components of modulus, the final relationship is obtained in equation 15. To the author's knowledge, such analytical relationship between two viscoelastic media does not exist in the literature.

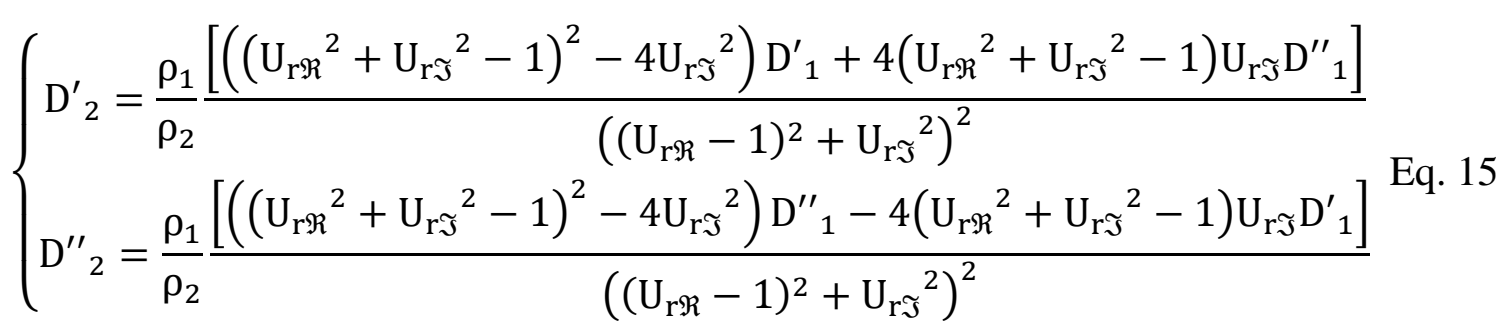




\section{UNCERTAINTY OF MEASURAND AND CHOICE OF REFERENCE}

\section{MATERIAL}

Equation 15 being strongly non-linear, the measurand error is also non-linear and can become detrimental for some material combinations. The choice of material 1 is therefore crucial to ensure that the mechanical properties of material 2 are measured with an acceptable error margin. The way to achieve a given measurement error on $\mathrm{U}_{\mathrm{r}}^{*}$ is not discussed in this paper. In this section, based on the literal expression of measurand error, guidelines to assist the selection of the reference material are provided.

In order to determine the error on the components of the module ( $\mathrm{D}^{\prime}$ and $\left.\mathrm{D}^{\prime \prime}\right)$, it is first necessary to estimate the error on the components of acoustic impedance (equation 16 obtained from equation 8).

$$
\left\{\begin{array}{c}
\mathrm{D}_{2}{ }^{\prime}=\left(\mathrm{Z}_{\mathfrak{R} 2}{ }^{2}-\mathrm{Z}_{\Im 2}{ }^{2}\right) / \rho_{2} \\
\mathrm{D}_{2}{ }^{\prime \prime}=2 \mathrm{Z}_{\mathfrak{R} 2} \mathrm{Z}_{\Im 2} / \rho_{2}
\end{array}\right.
$$

For the sake of simplicity, the error on the real and imaginary part of the reflected amplitude are set equal and noted $\Delta \mathrm{U}$. Arguments of $\mathrm{Z}_{1}{ }^{*}$ and $\mathrm{Z}_{2}{ }^{*}$ are respectively denoted $\theta_{1}$ and $\theta_{2}$. The acoustic impedance argument is equal, as usual, to half the angle of the loss factor $\delta_{2}\left(\tan \left(\delta_{2}\right)=\mathrm{D}_{2}{ }^{\prime \prime} / \mathrm{D}_{2}{ }^{\prime}\right)$.

By differentiating equation 16, we obtain

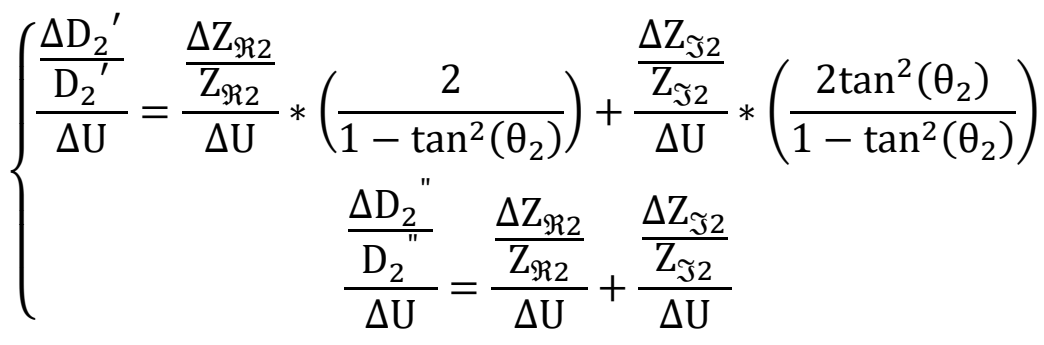

Where 


$$
\left\{\begin{array}{l}
\frac{\frac{\Delta \mathrm{Z}_{\Re 2}}{\mathrm{Z}_{\Re 2}}}{\Delta \mathrm{U}}=\left(\left|\frac{\partial \mathrm{Z}_{\Re 2}}{\partial \mathrm{U}_{\mathrm{r} \Re}}\right|+\left|\frac{\partial \mathrm{Z}_{\Re 2}}{\partial \mathrm{U}_{\mathrm{r} \mathfrak{I}}}\right|\right) * \frac{1}{\mathrm{Z}_{\Re 2}} \\
\frac{\Delta \mathrm{Z}_{\mathfrak{I} 2}}{\mathrm{Z}_{\Im 2}}=\left(\left|\frac{\partial \mathrm{Z}_{\mathfrak{I} 2}}{\partial \mathrm{U}}\right|+\left|\frac{\partial \mathrm{Z}_{\mathfrak{I} 2}}{\partial \mathrm{U}_{\mathrm{r} \mathfrak{R}}}\right|\right) * \frac{1}{\mathrm{U}_{\mathrm{r} \mathfrak{I}}}
\end{array}\right.
$$

Figure 4 represents the acoustical impedance moduli ratio which corresponds to the minimal error on the determination of $\mathrm{D}_{2}$ ' and $\mathrm{D}_{2}$ " for given values of impedance arguments of reference material $\left(\theta_{1}\right)$ and material to be characterised $\left(\theta_{2}\right)$ : (a) and (b) are respectively related to real and imaginary part of $\mathrm{D}_{2}{ }^{*}$. Impedance arguments vary in the whole physical domain from $0^{\circ}$ (purely elastic solid behaviour) to $45^{\circ}$.

It appears that, whatever the impedance arguments of materials 1 and 2 are, the optimum value of the moduli ratio is always in the range 0.6 to 1.6. To this optimum ratio corresponds a minimum error that is plotted in figure 5. This minimal error depends mainly on the impedance argument of medium 2 . The following analysis is based on the assumption of a measurement error of $1 \%$ on the reflected amplitude. In what concerns the real part of modulus $\mathrm{D}_{2}$, a minimal error in an acceptable range (i.e. $<10 \%$ ) is only obtained for an impedance argument of medium 2 lower than $25^{\circ}$. For the imaginary part of modulus $\mathrm{D}_{2}$ ", the impedance argument of medium 2 has to be higher than $14^{\circ}$. Hence, with an assumption on the measurement error of $1 \%$, both components of the modulus can only be determined accurately for an impedance argument in the range $14^{\circ}$ to $25^{\circ}$, which corresponds to $0.5<\tan \left(\delta_{2}\right)<1.2$. 


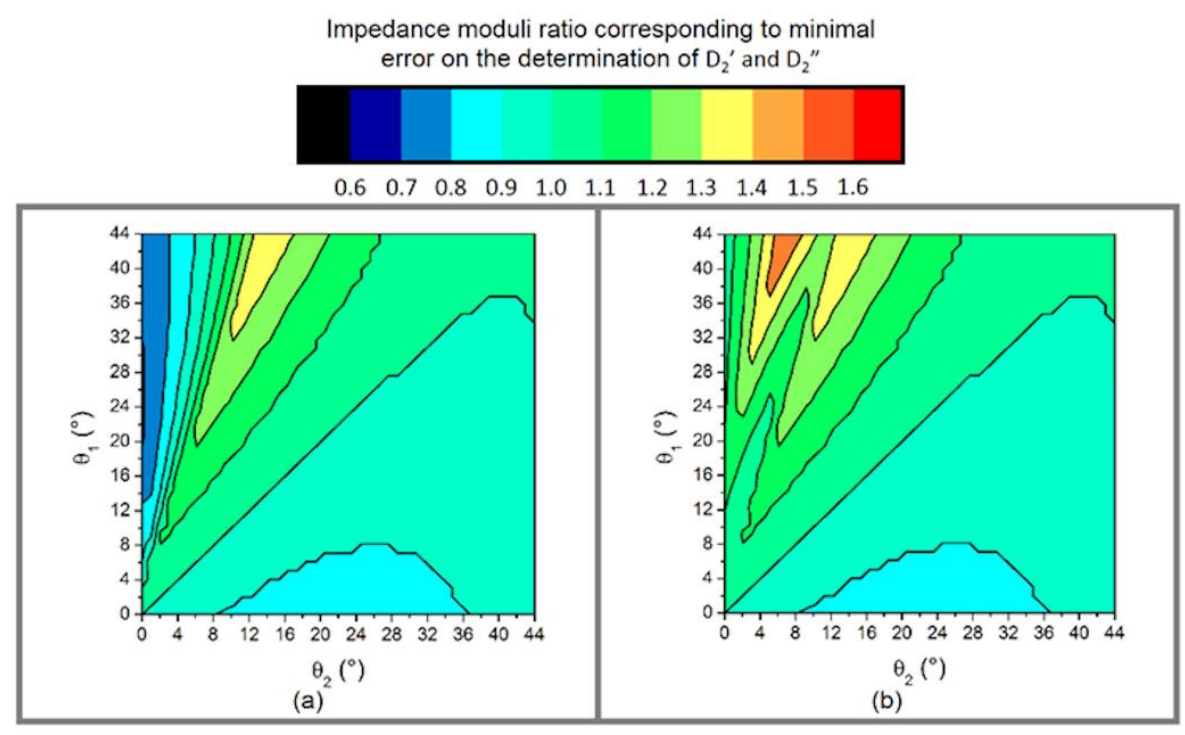

Figure 4. Impedance moduli ratio corresponding to minimal error on $\mathrm{D}_{2}$ ' (left) and $\mathrm{D}_{2}{ }^{\prime}$ (right) in function of impedance arguments of reference material $\left(\theta_{1}\right)$ and material to be characterised $\left(\theta_{2}\right)$ :

(a) Determination of $\mathrm{D}_{2}$ '

(b) Determination of $\mathrm{D}_{2}$ "

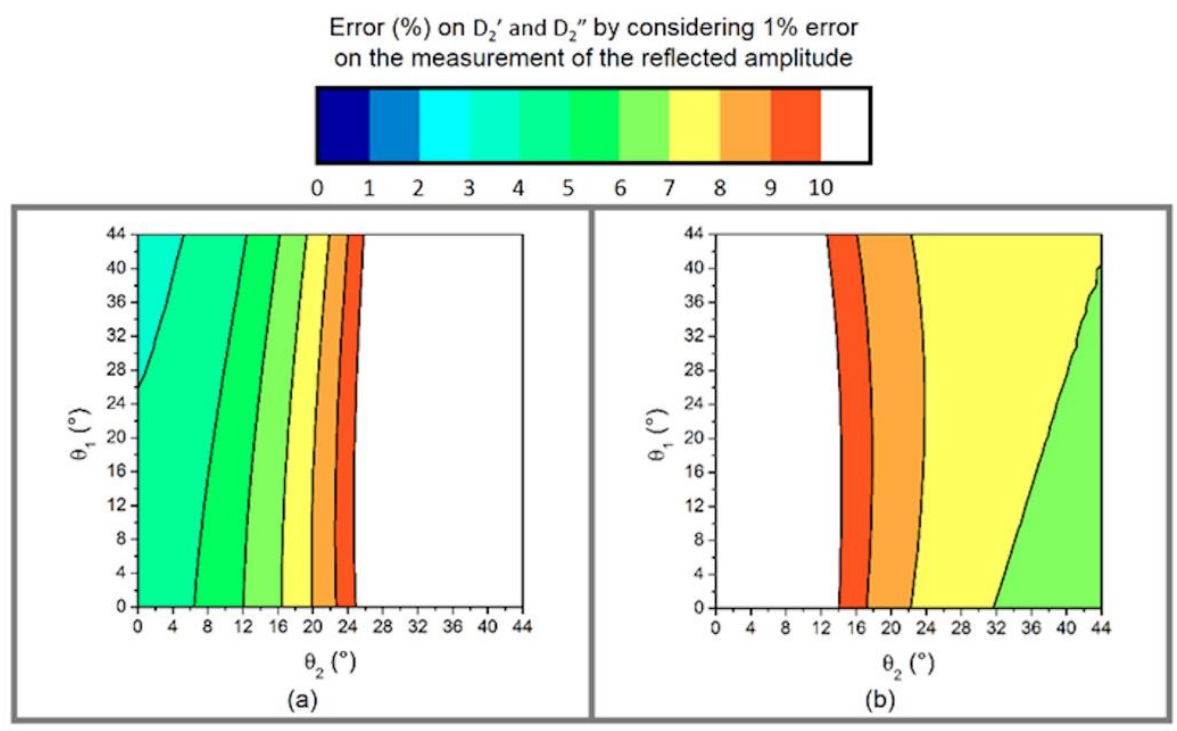


Figure 5. Minimal relative error in function of impedance arguments of reference material $\left(\theta_{1}\right)$ and material to be characterised $\left(\theta_{2}\right)$ :

(a) Determination of $\mathrm{D}_{2}$ '

(b) Determination of $\mathrm{D}_{2}$ "

Two limit cases will now be investigated: loss modulus of medium 1 is null $\left(\theta_{1}=0^{\circ}\right)$ as illustrated in figure 6 and storage modulus of medium 1 null $\left(\theta_{1}=45^{\circ}\right)$ as presented in figure 7.

Figures 6 and 7 represent the error in determining the real (a) and imaginary (b) part of the complex modulus of material 2 in function of $\theta_{2}$ and $\log _{10}\left(\left|Z_{2} / Z_{1}\right|\right)$. As in the previous case, let us consider a case in which the uncertainty of amplitude measurement can be estimated at $1 \%$ and the error is expected to be less than $10 \%$.

The frontier of the domain of acceptable error $(<10 \%)$ is symbolised by the thick black line. The fields of applicability of the method are similar for the two extreme values of $\theta_{1}$ considered. Whatever $\theta_{1}$ and the modulus component, the minimum error is in the vicinity of a moduli ratio of 1 , a result in accordance with previous findings (see figure 4).

Nevertheless, a reasonable accuracy is maintained over an extended moduli ratio range (0.1 to 10 ) if material 2 is purely linear elastic (only the real part of the complex modulus has to be determined in this case) or purely viscous (in which case only the imaginary part of the complex modulus has to be determined). However, in the case of a viscoelastic medium with an angle in a median zone, the choice of medium 1 is much more restricted to achieve the desired accuracy. For instance, let us consider a 
viscoelastic solid whose loss factor $\left(\tan \left(\delta_{2}\right)=\mathrm{D}_{2}{ }^{\prime \prime} / \mathrm{D}_{2}{ }^{\prime}\right)$ is of 1 . The corresponding impedance argument would be $\theta_{2}=\delta_{2} / 2=22.5^{\circ}$. In this case, a sufficiently accurate estimation of both D' and D" would only be obtained for an impedance module ratio between 0.5 and 2 .

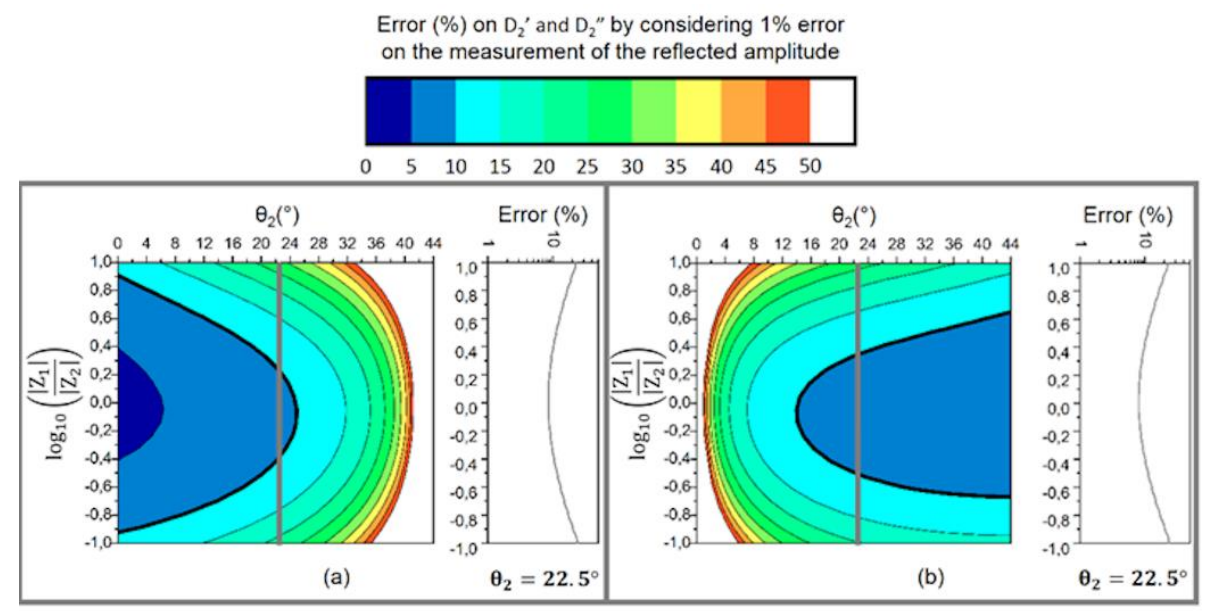

Figure 6. Error in determining the real (a) and imaginary (b) parts of the complex modulus of the material to be tested in function of moduli ratio when the reference material is purely elastic $\left(\theta_{1}=0^{\circ}\right)$. A profile with an isovalue of $\theta_{2}=22.5^{\circ}$ is represented on the right side of each plot.

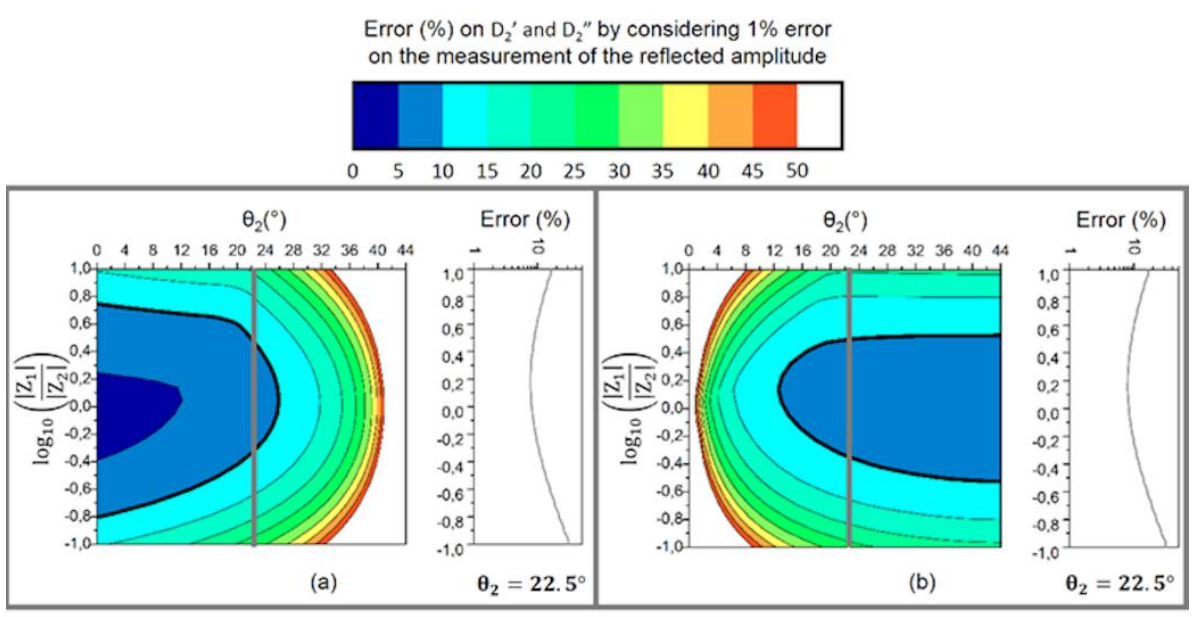


Figure 7. Error in determining the real (a) and imaginary (b) parts of the complex modulus of the material to be tested in function of moduli ratio when the reference material is purely viscous $\left(\theta_{1}=45^{\circ}\right)$. A profile with an isovalue of $\theta_{2}=22.5^{\circ}$ is represented on the right side of each plot.

The error study highlights that only the storage moduli ratio between the two contacting media had an impact on the accuracy of the measurand. From this point of view, $\mathrm{D}^{\prime \prime}{ }_{1}$ can take on any value. By contrast, a too large viscous component (which causes a very strong attenuation in the reference medium) would be detrimental in terms of the accuracy of the measurement of the reflected waves' amplitudes. In all cases, a high degree of accuracy in the measurement of reflected waves' amplitudes is required: in the most favourable case, the error on the modules is four times greater than that on amplitudes. More generally speaking, this method can be applied either for longitudinal or transverse waves. In the first case, media 1 and 2 can be either fluids or solids. In the second case, media 1 must be solid and media 2 can be either solid or fluid. In the last case, the applicability of the method implies high ultrasonic frequency and high viscosity so that the acoustical impedance of the fluid is equivalent to the one of a solid.

\section{DISCUSSION}

In the previous section, it is highlighted how to achieve the best measurand accuracy by applying the SRM. In the following, the accuracy obtained with this method is compared with that of traditional methods in order to determine the respective fields of application of the different methods. 
Traditional methods (transmission mode, transmission mode in water bath, pulse-echo mode) are based, on the one hand, on the measurement of a transit time and a sample thickness in order to deduce the wave celerity and, on the other hand, on amplitude ratio measurements to deduce the attenuation. Depending on the method, the amplitude ratio most commonly used is as follows:

- Transmission mode: ratio of wave amplitudes travelling through samples of different thicknesses

- Transmission mode in water bath: ratio of transmitted waves' amplitudes with and without sample

- Pulse-echo mode: ratio of successive echo amplitudes

The wave celerity $\mathrm{c}$ is calculated using equation 19 , where $\mathrm{d}$ represents the distance travelled by the wave and the corresponding transit time. The attenuation coefficient $\alpha$ is obtained from equation 20 , where $\mathrm{U}$ is the amplitude ratio determined according to the considered mode.

$$
\begin{aligned}
& \mathrm{c}=\mathrm{d} / \mathrm{t} \\
& \left\{\begin{array}{l}
\alpha=\ln (\mathrm{U}) / \mathrm{d} \text { for transmission and pulse }- \text { echo modes } \\
\alpha=\ln (\mathrm{U}) / \mathrm{d}+\alpha_{\mathrm{w}} \text { for transmission mode in water bath }
\end{array}\right.
\end{aligned}
$$

Based on equations 6 and 20, the error on $\mathrm{D}^{\prime}$ and $\mathrm{D}^{\prime \prime}$ is expressed in terms of the argument of the acoustic impedance of the tested material $\tan (\theta)$ and $u$ (equation 21) and is plotted in figure 8. Only the error associated with the determination of $U$ is taken into account. The error on $\mathrm{c}$ has been considered negligible: an error of less than $1 \%$ is easily achieved, which is small compared to other sources of error. 


$$
\left\{\begin{array}{c}
\frac{\frac{\Delta \mathrm{D}^{\prime}}{\mathrm{D}^{\prime}}}{\Delta \mathrm{U}}=-2 \tan ^{2}(\theta) \frac{3-\tan ^{2}(\theta)}{1-\tan ^{4}(\theta)} \frac{1}{\mathrm{U} \ln (\mathrm{U})} \\
\frac{\frac{\Delta \mathrm{D}^{\prime \prime}}{\mathrm{D}^{\prime \prime}}}{\Delta \mathrm{U}}=\left|\frac{1-3 \tan ^{2}(\theta)}{1+\tan ^{2}(\theta)} \frac{1}{\mathrm{U} \ln (\mathrm{U})}\right|
\end{array}\right.
$$

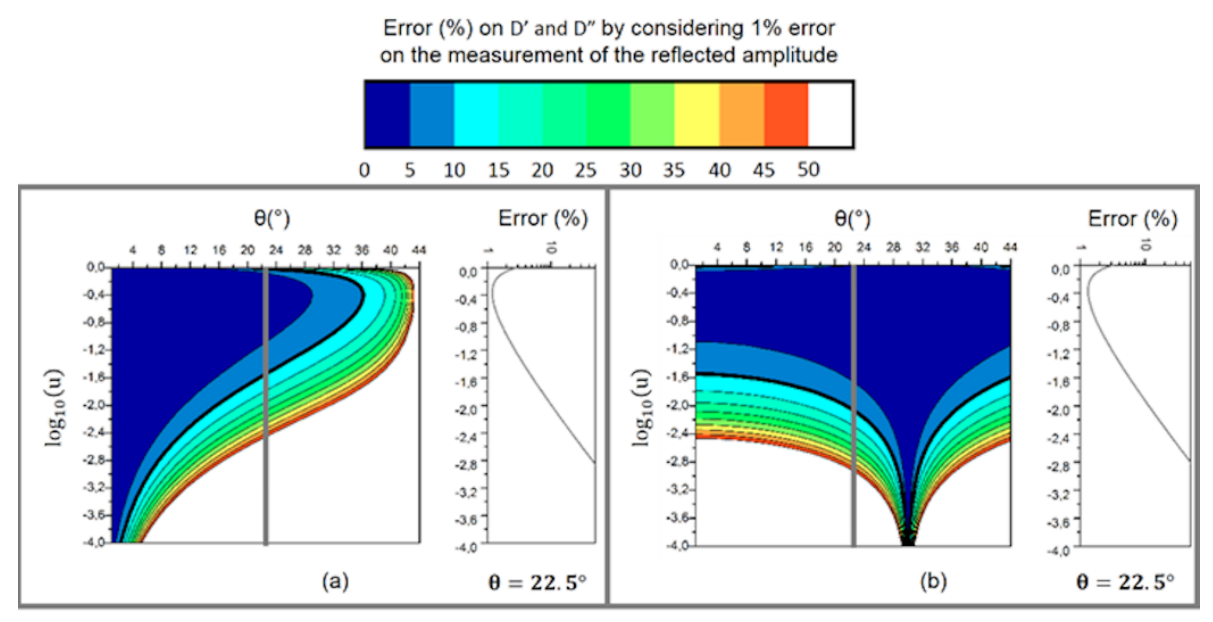

Figure 8. Error in determining the real (a) and imaginary (b) parts of the complex modulus of the material to be tested against amplitude ratio. A profile with an isovalue of $\theta=22.5^{\circ}$ is represented on the right side of each plot.

At a given angle, the minimum error is obtained for a value of $U=1 / \mathrm{e}=0.368$. Another noteworthy value is the angle $\theta=30^{\circ}$ for which the theoretical error on $D^{\prime \prime}$ is zero. More generally, an acceptable error (i.e. $<10 \%$ ) is only obtained for sufficiently high values of $U$. Considering the case of a solid with $\tan (\delta)=1$ (corresponding to an angle $\theta=22.5^{\circ}$ ), an error of less than $10 \%$ can only be obtained for a value of $\mathrm{U}$ greater than about 0.015 .

The conventional modes can be very accurate if the thickness of the samples can be adjusted to obtain a value of $U$ sufficiently close to the optimal value of 0.368 . Under 
specific conditions, the required sample thickness would be so small that it prevent the practical implementation of the method. For detailed demonstration, please refer to Part 2.

\section{CONCLUSION}

The aim of this paper is to highlight the potential scope of the Surface Reflection Method (SRM). First, analytical formulas were established to relate the mechanical characteristics of a sample to be tested to those of a reference sample as well as to the amplitude of the waves reflected at the interface. The error study demonstrated that, in order to obtain a given accuracy on the measurands, the moduli ratio of the acoustic impedances of the two materials in contact had to be close (typically between 0.6 and 1.6). These conditions for the validity of the method apply to both viscoelastic and considered elastic materials. Although the error on the measurand expected with conventional methods is generally lower than that of the SRM, the conditions required to obtain a given accuracy cannot be met in some cases such as the study of the mechanical properties of polymers in rubbery state or highly viscous fluids (honey, crude oil, colloid suspensions ...). For this type of tests, the only possible measurement method is the SRM. Other possible fields of application are quality control, the measurement of ageing or the study of the durability of materials.

\section{ACKNOWLEDGMENT}

The authors wish to thank: Pascale Lenig-Santoro for providing language help, Demathieu et Bard industry for their financial support, the National Center for Scientific 
Research (CNRS) and the Robert Schuman Institute of Technology, part of the University of Strasbourg, for their financial support.

This is a preprint of an article published in Experimental Mechanics. The final authenticated version is available online at: https://doi.org/ 10.1007/s11340-021-00730-

9.

\section{ETHICAL STATEMENT / CONFLICT OF INTEREST}

All authors certify that they have no affiliations with or involvement in any organization or entity with any financial interest or non-financial interest in the subject matter or materials discussed in this manuscript.

\section{REFERENCES}

[1] Ferry JD (1980) Viscoelastic properties of polymers. John Wiley \& Sons

[2] Truell R, Elbaum C, Chick BB (1969) Ultrasonic methods in solid state physics. Academic Press

[3] Nolle AW, Sieck PW (1952) Longitudinal and transverse ultrasonic waves in a synthetic rubber. J Appl Phys 23:888 - 893. https://doi.org/10.1063/1.1702325

[4] Fujisawa K, Takei Y (2009) A new experimental method to estimate viscoelastic properties from ultrasonic wave transmission measurements. J Sound Vib 323:609 - 625. https://doi.org/10.1016/j.jsv.2009.01.016

[5] Lillamand I, Chaix J-F, Ploix M-A, Garnier V (2010) Acoustoelastic effect in concrete material under uni-axial compressive loading. NDT\&E International 43:655-660. https://doi.org/10.1016/j.ndteint.2010.07.001

[6] Fan LF, Wu ZJ, Wan Z, Gao JW (2017) Experimental investigation of thermal 
effects on dynamic behaviour of granite. Appl Therm Eng 125:94 - 103. https://doi.org/10.1016/j.applthermaleng.2017.07.007

[7] Espinosa L, Prieto F, Brancheriau L, Lasaygues P (2019) Effect of wood anisotropy in ultrasonic wave propagation: A ray-tracing approach. Ultrasonics 91:242 - 251. https://doi.org/10.1016/j.ultras.2018.07.015

[8] Tinard V, Brinster M, Francois P, Fond C (2018) Experimental assessment of sound velocity and bulk modulus in high damping rubber bearings under compressive loading. Polym Test 65:331 - 338 . https://doi.org/10.1016/j.polymertesting.2017.12.010

[9] Ivey DG, Mrowca BA, Guth E (1949) Propagation of ultrasonic bulk waves in high polymers. J Appl Phys 20:486 - 492. https://doi.org/10.1063/1.1698415

[10] Kono R (1960) The dynamic bulk viscosity of polystyrene and polymethyl methacrylate. J Phys Soc Jpn 15:718 - 725. https://doi.org/10.1143/JPSJ.15.718

[11] Capps RN (1985) Influence of carbon black fillers on acoustic properties of polychloroprene (neoprene) elastomers. J Acoustic Soc Am 78:406 - 413. https://doi.org/10.1121/1.392462

[12] Wu J (1996) Determination of velocity and attenuation of shear waves using ultrasonic spectroscopy. J Acoustic Soc Am 99:2871 - 2875. https://doi.org/10.1121/1.414880

[13] Paterson DAP, Ijomah W, Windmill JFC (2018) Elastic constant determination of unidirectional composite via ultrasonic bulk wave through transmission measurements: A review. Prog Mater Sci 97:1 - 37. https://doi.org/10.1016/j.pmatsci.2018.04.001 
[14] McSkimin HJ, Andreatch P (1962) Analysis of the pulse superposition method for measuring ultrasonic wave velocities as a function of temperature and pressure. $\mathbf{J}$ Acoustic Soc Am 34:609 - 615. https://doi.org/10.1121/1.1918175

[15] Bastien P (1977) The possibilities and limitations of ultrasonics in the nondestructive testing of steel. NDT International $297-305$. https://doi.org/10.1016/0308-9126(77)90003-7

[16] Afifi H, Marzouk S, Abd el Aal N (2007) Ultrasonic characterization of heavy metal TeO2 - WO3 - PbO glasses below room temperature. Physica B: Physics of Condensed Matter 390:65 - 70. https://doi.org/10.1081/PPT-120017922

[17] Hagan CP, Orr JF, Mitchell CA, Dunne NJ (2015) Critical evaluation of pulseecho ultrasonic test method for the determination of setting and mechanical properties of acrylic bone cement: Influence of mixing technique. Ultrasonics 56:279 - 286. https://doi.org/10.1016/j.ultras.2014.08.008

[18] Metwally K, Lefevre E, Baron C, et al (2016) Measuring mass density and ultrasonic wave velocity: a wavelet-based method applied in ultrasonic reflection mode. Ultrasonics 65:10 - 17. https://doi.org/10.1016/j.ultras.2015.09.006

[19] Burg EVD, Grill W (2010) Characterization of elastomers with transverse sonic waves. Polym Test 29:281 - 287 . https://doi.org/10.1016/j.polertesting.2009.12.001

[20] Mason WP, Baker WO, McSkimin HJ, Heiss JH (1949) Measurement of shear elasticity and viscosity of liquids at ultrasonic frequencies. Phys Rev 75:936 - 946. https://doi.org/10.1103/PhysRev.75.936

[21] O’Neil HT (1949) Reflection and refraction of plane shear waves in viscoelastic media. Phys Rev 75:928 - 935. https://doi.org/10.1103/PhysRew.75.928 
[22] Yoneda A, Ichibara M Shear viscoelasticity of ultrasonic couplers by broadband reflectivity measurements. J Appl Phys 97:2005.

https://doi.org/10.1063/1.1850180

[23] Alig I, Sulimma J, Tadjbakhsch S (1997) Ultrasonic shear wave reflexion method for measurements of the viscoelastic properties of polymer films. Rev Sci Instrum 68:1536 - 1542. https://doi.org/10.1063/1.1147643

[24] Chang J-J, Li Y-Y, Zeng X-F, et al (2019) Study on the viscoelasticity measurement of materials based on surface reflected waves. Materials 12:18751890. https://doi.org/10.1063/1.4918787

[25] Omata N, Suga T, Furusawa H, et al (2006) Viscoelasticity evaluation of rubber by surface reflection of supersonic wave. Ultrasonics 44:211-215. https://doi.org/10.1016/j.ultras.2006.06.019

[26] Kinsler LE, Frey AR, Coppens AB, Sanders JV (2000) Fundamentals of Acoustics. John Wiley \& Sons

[27] Lockett FJ (1962) The reflection and refraction of waves at an interface between viscoelastic materials. J Mech Phys Solids 10:53 - 64. https://doi.org/10.1016/0022-5096(62)90028-5

[28] Cooper HF (1967) Reflection and transmission of oblique plane waves at a plane interface between viscoelastic media. J Acoustic Soc Am 42:1064 - 1069. https://doi.org/10.1121/1.1910691

[29] Schoenenberg M (1971) Transmission and reflection of plane waves at an elasticviscoelastic interface. Geophys J Royal Astronom Soc 25:35 - 47. https://doi.org/10.1111/j.1365-246X.1971.tb02329.x 
